\title{
RECURRENT AND POISSON STABLE FLOWS ${ }^{1}$
}

\author{
RONALD A. KNIGHT
}

\begin{abstract}
The purpose of this paper is to demonstrate that the families of Poisson stable flows and recurrent flows coincide whenever the phase space is locally compact.
\end{abstract}

1. Introduction. The concepts of recurrent motions, nonwandering points, Poisson stable points, and minimal sets are classical notions central to the qualitative theory of motions for dynamical systems. Birkhoff introduced recurrent motions and nonwandering points (see [6]) and established important interrelationships for recurrent motions, minimal sets, nonwandering points, and Poisson stability for certain $n$-manifold regions. Most of these results have since been extended to Hausdorff or locally compact Hausdorff phase spaces. Our principal task in this paper is to demonstrate that the notions of recurrence and Poisson stability coincide in certain flows. Moreover, we give examples to show that the results are sharp.

Throughout the paper we shall assume that there is a given flow $(X, \pi)$ on a Hausdorff phase space $X$. We denote the orbit, orbit closure, limit set, prolongation, and prolongational limit set relations on $X$, respectively, by $C, K, L, D$, and $J$. The unilateral versions of these relations carry the appropriate + or - superscript. A point $x$ in $X$, motion $\pi_{x}$, and trajectory $C(x)$ are called recurrent if and only if, given any neighborhood $U$ of $x$, there is a $T \geqslant 0$ such that $U \cap y[0, T] \neq \varnothing$ for every $y \in C(x)$. A point $x$ in $X$ is said to be positively (negatively) Poisson stable provided $x \in L^{+}(x)\left(x \in L^{-}(x)\right)$ and $x$ is said to be Poisson stable if it is both positively and negatively Poisson stable. A point $x$ in $X$ is nonwandering if $x \in J^{+}(x)$, or equivalently, $x \in J^{-}(x)$. If one of the properties above holds at each point of the phase space, then the flow is said to have that property. A set $M \subset X$ is called (positively, negatively) minimal if and only if it is a closed (positively, negatively) invariant set containing no nonempty proper subset with these properties. The closure of the set of Poisson stable points is called the center of the flow or the set of central motions. The flow $(X, \pi)$ is said to be central if $X$ is the set of central motions. A set $M \subset X$ is said to be positively (negatively) stable provided each neighborhood $U$ of $M$ contains a neighborhood $V$ such that $C^{+}(V) \subset U\left(C^{-}(V) \subset U\right)$. A set $M \subset X$ is called a saddle set if and only if there

Received by the editors March 10, 1980 and, in revised form, July 18, 1980.

AMS (MOS) subject classifications (1970). Primary 34C35; Secondary 54H20.

Key words and phrases. Center, central motion, characteristic $0^{ \pm}$, compact minimal set, continuous flow, dynamical system, nonwandering, orbit space, Poisson stable, recurrent motion, saddle set, stable.

${ }^{1}$ This research was supported by an NMSU faculty research grant. 
is a neighborhood $U$ of $M$ such that every neighborhood $V$ of $M$ contains a point $x$ with $C^{+}(x) \not \subset U$ and $C^{-}(x) \not \subset U$. A flow $(X, \pi)$ is said to be of characteristic $0^{+}$ $\left(0^{-}\right)$provided $D^{+}(x)=K^{+}(x)\left(D^{-}(x)=K^{-}(x)\right)$ for every $x$ in $X$ and to be of characteristic $0^{ \pm}$if both conditions hold (see [1]). Finally, $(X, \pi)$ is called dispersive if $J(x)=\varnothing$ for each $x$ in $X$.

We denote the closure, boundary, and complement of a set $M \subset X$ by $\bar{M}, \partial M$, and $X \backslash M$, respectively. The sets of Poisson stable points and nonwandering points are denoted by $P_{s}$ and $W$, respectively. The set of nonnegative (nonpositive) reals is denoted by $R^{+}\left(R^{-}\right)$.

The reader may consult references [2] through [5] for the basic dynamical system concepts used herein.

2. Recurrence and Poisson stability. Each point of a compact minimal set is recurrent $[3,4.22]$ and, of course, equal to the orbit closure of each of its points. Conversely, whenever the phase space is locally compact, the orbit closure of each recurrent point is compact minimal $[3,4.24]$. These properties were first demonstrated by Birkhoff for regions of $n$-manifolds [6]. Bhatia and Hajek show that, for a locally compact phase space, an orbit closure $K(x)$ is compact minimal if and only if each of its points is attracted to $x$, i.e. $x \in L^{+}(y)$ for each $y \in K(x)$. The compact minimality, recurrence, and Poisson stability concepts on a flow are equivalent as the following theorem indicates.

THEOREM 1. A flow on a locally compact space $X$ is recurrent if and only if it is (positively, negatively) Poisson stable.

Proof. First, let $(X, \pi)$ be Poisson stable and let $x$ be a point of $X$. We shall proceed by showing that $x \in L^{+}(y)$ for each $y \in L^{+}(x)$. Suppose the contrary. Let $y$ be a point of $L^{+}(x)$ such that $x \notin L^{+}(y)$. Select relatively compact open neighborhoods $V$ and $U$ of $x$ such that $\bar{V} \subset U$ and $K(y)=L^{+}(y) \subset X \backslash \bar{U}$. The neighborhood $\bar{U}$ can be chosen so that it contains no complete orbit. For if this were impossible, then each closed neighborhood $G$ of $x$ would contain a complete orbit $C\left(x_{G}\right)$ with $x_{G} \rightarrow x$. Moreover, since $x$ is not critical or periodic, we can select a closed neighborhood $G_{0}$ of $x$ and a $t>0$ such that $x t \notin G_{0}$, and hence, we have the absurdity $x_{G} t \rightarrow x t \in G_{0}$. Next, let

$$
t_{z}=\inf \left\{t \in R^{+}: z t \in U \text { and } z[0, t] \cap(X \backslash \bar{U}) \neq \varnothing\right\}
$$

for each $z \in U$. Since each point is Poisson stable, each $z \in \bar{V}$ has a neighborhood $V_{z} \subset U$ such that $V_{z} t \subset X \backslash \bar{U}$ and $V_{z} t^{\prime} \subset U$ for some $t$ and $t^{\prime}$ with $0<t<t^{\prime}$. Also, we can select $t_{0}$ so that $t_{p} \leqslant t_{0}<t_{z}+1$ for each $p \in V_{z}$. Since $\bar{V}$ is compact and $\left\{V_{z}: z \in \bar{V}\right\}$ covers $\bar{V}$, there is a finite subcover $\left\{V_{z_{1}}, \ldots, V_{z_{n}}\right\}$ for $\bar{V}$. Thus,

$$
T=\sup \left\{t_{z}: z \in \bar{V}\right\} \leqslant \max \left\{t_{z_{i}}+1: 1<i<n\right\}<+\infty \text {. }
$$

Now, select a net $\left(t_{i}\right)$ such that $x t_{i} \rightarrow y$ and $t_{i} \rightarrow+\infty$. Let $T_{i}=\max \left\{t \in R^{+}\right.$: $x t \in \partial V$ and $\left.t \leqslant t_{i}\right\}$. Some subnet $\left(x T_{m_{i}}\right)$ of $\left(x T_{i}\right)$ converges to a point $z$ in the compact set $\partial V$. Ultimately, the net $\left(x t_{n_{i}}\right)$ is in $X \backslash \bar{U}$, and hence, for some $i_{0}$ we 
have $x\left(T_{n_{i}}, t_{n_{i}}\right] \subset X \backslash \bar{V}$ for $i \geqslant i_{0}$. Also, $x t_{n_{i}}=\left(x T_{n_{i}}\right)\left(t_{n_{i}}-T_{n_{i}}\right) \in \partial V[0, T]$ for $i \geqslant$ $i_{0}$ which yields $y \in \partial V[0, T]$. Thus, $C^{-}(y) \cap \bar{V} \neq \varnothing$ which contradicts $C(y) \subset$ $K(y)=L^{+}(y) \subset X \backslash \bar{U}$. Whence, we have $y \in L^{+}(x)$ and $L^{+}(y)=L^{+}(x)$.

Each point of $K(x)=L^{+}(x)$ is weakly attracted to $x$, i.e. $x \in L^{+}(y)$ for each $y \in K(x)[3,2.4 .2]$. Bhatia and Hajek have shown that for $X$ locally compact, each point of $K(x)$ is weakly attracted to $x$ if and only if $K(x)$ is compact minimal [4, 4.6]. Thus, $L^{+}(x)$ is compact minimal in $X$ for each $x \in X$. Each point of a compact minimal set is recurrent $[3,4.22]$. Consequently, $X$ is recurrent.

The converse is a known result for any Hausdorff phase space $X[3,4.10 .2]$ completing the proof of the theorem.

COROLlaRY 1.1. Let $(X, \pi)$ be locally compact. Then the following are equivalent for a closed invariant set $M$.

(a) $M$ is compact minimal;

(b) $M$ is compact positively (negatively) minimal;

(c) $M$ is a (positively, negatively) Poisson stable orbit closure; and

(d) $M$ is an orbit closure, each point of which is recurrent.

Proof. The equivalence of (a), (b), and (d) is known [3, 4.18, 4.19, 4.22, 4.24]. The remainder of the result follows by Theorem 1 .

In view of the extension of Birkhoff's central motion theorem [9, 5.08] to locally compact spaces in [1], [8], we have an additional corollary.

COROLlARY 1.2. Let $X$ be locally compact. The nonwandering points are recurrent if and only if the set of Poisson stable points is closed.

Since every orbit closure in a locally compact space is minimal if and only if each of its points is recurrent, we now have equivalence of the concepts of minimal limit set, recurrent limit set, and (positively, negatively) Poisson stable limit set. On the other hand, a (positively, negatively) Poisson stable point need not have a limit set each of whose points is (positively, negatively) Poisson stable or recurrent. Nemytskii and Stepanov define a flow on the torus $T$ with exactly one critical point $p$, every other trajectory of which is dense in $T[9,4.06]$. For one orbit $C(x)$ and one orbit $C(y)$ we have $L^{-}(x)=L^{+}(y)=\{p\}$ and $L^{+}(x)=L^{-}(y)=T$. Every other noncritical orbit $C(z)$ satisfies $L^{+}(z)=L^{-}(z)=T$. Thus, the torus is the limit set of both positively and negatively Poisson stable points but only the critical point is recurrent. When $X$ is not locally compact Theorem 1 need not be valid. For example, let $X$ consist of exactly one noncritical trajectory of the toral flow and restrict the flow to $X$. Then $(X, \pi)$ may be chosen either positively Poisson stable, negatively Poisson stable, or both, but no point of $X$ is recurrent. If $X$ is selected to include the critical point and one orbit bilaterally dense in the torus, then $X$ is Poisson stable at each point but is neither recurrent nor minimal.

Using the Ura-Kimura alternatives [2,9.1], we obtain the following condition for a compact invariant set $M$ in a locally compact phase space concerning the properties: $M$ is (a) positively or negatively stable, (b) positively or negatively attracting, (c) a saddle set, and (d) not isolated from compact invariant sets. If $X$ is 
locally compact and $M$ is a compact invariant set, then $M$ may satisfy any combination of the properties (a) through (d) except those satisfying both (a) and (b). Of course, the only way properties (b) and (d) can be realized simultaneously is for $M$ to be a component of $X$. Similarly, if both parts of (a) hold when (b) holds or (d) fails, then $M$ is a component of $X$. Thus, if $M$ is not a component of $X$, then it may be positively or negatively stable, a positive or negative attractor, positively or negatively asymptotically stable, a saddle set, or a positive or negative saddle attractor with isolation from compact minimal sets possible or not whenever stability fails.

The succeeding proposition follows easily.

Proposition 2. Let $X$ be locally compact and $(X, \pi)$ be Poisson stable. Then each limit set is either bilaterally stable or a saddle set.

Corollary 2.1. If $X$ is locally compact and $(X, \pi)$ is Poisson stable, then the flow is of characteristic $0^{ \pm}$if and Qnly if each limit set is bilaterally stable.

If $X$ is locally compact and $x$ is neither periodic nor critical, then $\overline{L(x) \backslash C(x)}=$ $L(x)[3,4.5]$. Thus, in a Poisson stable flow a point $x$ is periodic or critical if and only if $L(x)=C(x)$. In [7] it is shown that $K(x)=C(x)(D(x)=C(x))$ for each $x \in X$ if and only if the orbit space $X / C$ is a $T_{1}$ (Hausdorff) space. Hence, by applying Ura's prolongational stability theorem we have

Proposition 3. A Poisson stable flow on a locally compact phase space $X$ is periodic if and only if the orbit space $X / C$ is $T_{1}$. Moreover, the flow is periodic with each orbit bilaterally stable if and only if the orbit space $X / C$ is Hausdorff.

The succeeding two theorems characterize the nonwandering flows in terms of orbital stability.

TheOREM 4. Let $X$ be locally compact and $X^{*}$ be its one point compactification. $A$ necessary and sufficient condition for $(X, \pi)$ to be central is that the center of the extended flow $\left(X^{*}, \pi^{*}\right)$ be bilaterally stable in $X^{*}$.

Proof. We shall denote properties relative to $X^{*}$ by affixing an asterisk. The center $P_{s}^{*}$ of the extended flow is $P_{s} \cup\{\infty\}$. Let $P_{s}^{*}$ be bilaterally stable in $X^{*}$. Then, by Ura's prolongational stability theorem, $D_{*}^{ \pm}\left(P_{s}^{*}\right)=P_{s}^{*}$, so that for $x \in$ $X^{*} \backslash P_{s}^{*}=W, D_{*}^{ \pm}(x) \subset W[2,7.6]$. Thus, $\infty \notin D_{*}^{ \pm}(x)$ and $D_{*}^{ \pm}(x)=D^{ \pm}(x)$. The sets $D^{+}(x)$ and $D^{-}(x)$ are compact yielding $L^{+}(x)$ and $L^{-}(x)$ nonempty. This means that $\varnothing \neq L(x) \subset D^{+}(x) \cup D^{-}(x) \subset W$, which is absurd. Whence, $X=\bar{P}_{s}$. The converse is obvious.

THEOREM 5. A flow is nonwandering if and only if its restiction to the wandering set is nondispersive of characteristic $0^{ \pm}$.

Proof. Assume that the restricted flow $(W, \pi \mid W)$ of the flow $(X, \pi)$ is nondispersive and let $x$ be a point of $W$ for which $J_{W}(x) \neq \varnothing$. Since $L_{W}(x) \subset L(x) \subset$ $X \backslash W$, we must have $L_{W}(x)=\varnothing$. Thus, $D_{W}^{ \pm}(x)=K_{W}^{ \pm}(x)=C^{ \pm}(x)$. Also, because 
$J_{W}(x) \neq \varnothing$ we have $J_{W}^{ \pm}(x)=C^{ \pm}(x)$. Thus, $x \in J_{W}(x) \subset J(x)$, implying that $x \in$ $X \backslash W$, contradicting $x \in W$. Hence, $W=\varnothing$ and $(X, \pi)$ is nonwandering. The converse follows trivially.

\section{REFERENCES}

1. S. Ahmad, Strong attraction and classification of certain contimuous flows, Math. Systems Theory 5 (1971), 157-163. MR 46 \#4507.

2. N. Bhatia and O. Hajek, Local semi-dynamical systems, Lecture Notes in Math., vol. 90, SpringerVerlag, Berlin and New York, 1969. MR 40 \#4559.

3. _ Theory of dynamical systems, Part I, Tech. Note BN-599, Univ. of Maryland, College Park, Md., 1969.

4. __ Theory of dynamical systems, Part II, Tech. Note BN-606, Univ. of Maryland, College Park, Md., 1969.

5. N. Bhatia and G. Szego, Stability theory of dynamical systems, Springer-Verlag, Berlin and New York, 1970.

6. G. Birkhoff, Dynamical systems, Amer. Math. Soc. Colloq. Publ., vol. 9, Amer. Math. Soc., Providence, R. I., 1966.

7. O. Hajek, Prolongations in topological dynamics, Seminar on Differential Equations and Dynamical Systems. II, Lecture Notes in Math., vol. 144, Springer-Verlag, Berlin and New York, 1970, pp. 79-89.

8. R. Knight, The center of a transformation group, Nonlinear Phenomena in Mathematical Sciences, edited by Lakshmikantham, (Proc. Internat. Conf. Nonlinear Phenomena in Math. Sci., Univ. of Texas, Arlington, June, 1980), Academic Press, New York (to appear).

9. V. Nemytskii and V. Stepanov, Qualitative theory of differential equations, English Transl., Princeton Univ. Press, Princeton, N. J., 1960.

Mathematics Division, Northeast Missouri State University, Krrksville, Missouri 63501 